\title{
A mixed spectral method for incompressible viscous fluid flow in an infinite strip*
}

\author{
WANG ZHONG-QING and GUO BEN-YU \\ Department of Mathematics, Shanghai Normal University \\ Division of Computational Science of E-Institute of Shangai Universities \\ Guilin Road 100, Shanghai, China, 200234 \\ E-mail: zqwang@shnu.edu.cn / byguo@shnu.edu.cn
}

\begin{abstract}
This paper considers the numerical simulation of incompressible viscous fluid flow in an infinite strip. A mixed spectral method is proposed using the Legendre approximation in one direction and the Legendre rational approximation in another direction. Numerical results demonstrate the efficiency of this approach. Some results on the mixed Legendre-Legendre rational approximation are established, from which the stability and convergence of proposed method follow.
\end{abstract}

Mathematical subject classification: 65M70, 35Q30, 41A10, 41A20.

Key words: incompressible viscous fluid flow, mixed Legendre-Legendre rational spectral method, infinite strip.

\section{Introduction}

Spectral methods have been used successfully for numerical solutions of differential equations, due to their high accuracy, see, e.g., $[2,5,6,7,9,10]$. The usual spectral methods are available only for bounded domains. However it is

\#614/04. Received: 30/VIII/04. Accepted: 05/IV/05.

*The work of these authors is supported in part by NSF of China, N.10471095, The Science Foundation of Shanghai N.04JC14062, The Special Funds for Doctorial Authorities of Chinese Education Ministry N.20040270002, The E-institutes of Shanghai Municipal Education Commission, N.E03004, The Special Funds for Major Specialities and The Fund N.04DB15 of Shanghai Education Commission. 
also important to consider spectral methods for unbounded domains. Recently, some spectral methods for unbounded domains were proposed, for instance, the Hermite and Laguerre spectral methods, see [8, 11, 17, 23, 26, 29]. By using these methods, we could approximate various differential equations directly. Indeed the weight functions $e^{-x}$ and $e^{-x^{2}}$ used in these approximations are too strong for some practical problems. We may also reformulate original problems in unbounded domains to singular problems in bounded domains by variable transformations, and then solve the resulting problems by the Jacobi spectral method, see [12-15]. In this case, we can use more suitable weight functions and obtain reasonable numerical results oftentimes. However, it is not easy to generalize this approach to multiple-dimensional problems. Another effective method is based on rational approximations, see, e.g., [3, 4, 18, 19, 20, 32]. So far, all of existing work is only for differential equations of second order.

This paper is devoted to the mixed Legendre-Legendre rational spectral method for the Navier-Stokes equation in an infinite strip. As is well known, this equation plays an important role in studying incompressible viscous fluid flow, see $[25,31]$. We usually consider the primitive equation with the velocity $\mathbf{u}$ and the pressure $p$. It is difficult to construct the base functions with free-divergence in spectral methods, and impossible to deal with the boundary values of pressure exactly. Therefore, it seems reasonable to construct numerical schemes based on certain alternative formulations of the Navier-Stokes equation. Some authors have used the vorticity-stream function form, see [25, 27]. However, there is no physical boundary condition on the vorticity. This fact always brings troubles in actual computation. Another way is to consider the stream function form as in $[16,23]$, in which the incompressibility is fulfilled automatically and the pressure does no longer appear. Moreover, it keeps the physical boundary conditions on the stream function. Thus this form is more appropriate for numerical simulation.

In this work, we shall approximate the stream function form of the NavierStokes equation in an infinite strip by using the Legendre approximation in one direction, and the Legendre rational approximation in another direction. This method has several advantages. Firstly, unlike the Jacobi approximation, we approximate the Navier-Stokes equation directly. Next, we can use the existing code of the Legendre approximation and so save a lot of work. Thirdly, we 
use the orthogonal approximation with the Legendre weight function as in the original problem, and so the numerical solution has some conservation properties as in the continuous case. This feature also simplifies actual computation and theoretical analysis.

This paper is organized as follows. In the next section, we propose the mixed Legendre-Legendre rational spectral scheme for the stream function form of Navier-Stokes equation, and present the main results on its stability and convergence. We also present some numerical results demonstrating the spectral accuracy of this method in the spatial variables. In section 3, we first establish some basic results on the mixed Legendre-Legendre rational approximation, which plays important role in numerical analysis of the related mixed spectral methods for differential equations of fourth order in an infinite strip. Then we prove the stability and convergence of the proposed scheme. The final section gives some concluding remarks.

\section{Mixed Legendre-Legendre Rational Spectral Method}

In this section, we first propose the mixed Legendre-Legendre rational approximation, and then construct a mixed scheme for the stream function form of Navier-Stokes equation. We state the results on the stability and convergence of the proposed scheme. We also present some numerical results showing the efficiency of this new approach.

\subsection{Mixed orthogonal approximation}

We first recall the Legendre approximation. Let $I=\{y|| y \mid<1\}$ and $\chi(y)$ be a certain weight function in the usual sense. Denote by $\mathbb{N}$ the set of all nonnegative integers. For any $r \in \mathbb{N}$, we define the weighted Sobolev space $H_{\chi}^{r}(I)$ in the usual way, and denote its inner product, semi-norm and norm by $(u, v)_{r, \chi, I},|v|_{r, \chi, I}$ and $\|v\|_{r, \chi, I}$, respectively. In particular, $L_{\chi}^{2}(I)=H_{\chi}^{0}(I)$, $(u, v)_{\chi, I}=(u, v)_{0, \chi, I}$ and $\|v\|_{\chi, I}=\|v\|_{0, \chi, I}$. For any $r>0$, we define $H_{\chi}^{r}(I)$ and its norm by interpolation as in [1]. The space $H_{0, \chi}^{r}(I)$ stands for the closure in $H_{\chi}^{r}(I)$ of the set $\mathcal{D}(I)$ consisting of all infinitely differentiable functions with compact support in $I$. When $\chi(y) \equiv 1$, we omit $\chi$ in the notations as usual. 
The Legendre polynomials $L_{l}(y)$ are the eigenfunctions of the singular SturmLiouville problem

$$
\partial_{y}\left(\left(1-y^{2}\right) \partial_{y} L_{l}(y)\right)+\lambda L_{l}(y)=0, y \in I, l=0,1,2 \cdots .
$$

The corresponding eigenvalues $\lambda=l(l+1)$. They satisfy the following recurrence relations

$$
\begin{gathered}
L_{l+1}(y)=\frac{2 l+1}{l+1} y L_{l}(y)-\frac{l}{l+1} L_{l-1}(y), l \geq 1, \\
(2 l+1) L_{l}(y)=\partial_{y} L_{l+1}(y)-\partial_{y} L_{l-1}(y), l \geq 1 .
\end{gathered}
$$

The set of Legendre polynomials is the complete $L^{2}(I)$-orthogonal system, namely,

$$
\int_{I} L_{l}(y) L_{m}(y) d y=\left(l+\frac{1}{2}\right)^{-1} \delta_{l, m}
$$

where $\delta_{l, m}$ is the Kronecker symbol. By virtue of (2.1) and (2.4),

$$
\int_{I} \partial_{y} L_{l}(y) \partial_{y} L_{m}(y)\left(1-y^{2}\right) d y=l(l+1)\left(l+\frac{1}{2}\right)^{-1} \delta_{l, m} .
$$

For any $N \in \mathbb{N}, \mathcal{P}_{N}$ stands for the set of all algebraic polynomials of degree at most $N$. Moreover,

$$
\begin{aligned}
{ }_{00} \mathcal{P}_{N} & =\left\{v \mid v \in \mathcal{P}_{N}, v(-1)=\partial_{y} v(-1)=0\right\}, \\
\mathcal{P}_{N}^{0,0} & =\left\{v \mid v \in \mathcal{P}_{N}, v( \pm 1)=\partial_{y} v( \pm 1)=0\right\} .
\end{aligned}
$$

In actual computation and numerical analysis, we need two specific Jacobi orthogonal projections. Let $\chi^{(\alpha, \beta)}(y)=(1-y)^{\alpha}(1+y)^{\beta}, \alpha, \beta>-1$ and

$$
{ }_{00} H_{\chi^{(\alpha, \beta)}}^{2}(I)=\left\{v \mid v \in H_{\chi^{(\alpha, \beta)}}^{2}(I) \text { and } v(-1)=\partial_{y} v(-1)=0\right\} .
$$

The orthogonal projection ${ }_{00} \tilde{P}_{N, \alpha, \beta}^{2}:{ }_{00} H_{\chi^{(\alpha, \beta)}}^{2}(I) \rightarrow{ }_{00} \mathcal{P}_{N}$ is defined by

$$
\left(\partial_{y}^{2}\left({ }_{00} \tilde{P}_{N, \alpha, \beta}^{2} v-v\right), \partial_{y}^{2} \phi\right)_{\chi}^{(\alpha, \beta)}, I=0, \quad \forall \phi \in{ }_{00} \mathcal{P}_{N} .
$$

We also define the orthogonal projection $\tilde{P}_{N, \alpha, \beta}^{2,0}: H_{0, \chi^{(\alpha, \beta)}}^{2}(I) \rightarrow \mathcal{P}_{N}^{0,0}$, by

$$
\left(\partial_{y}^{2}\left(\tilde{P}_{N, \alpha, \beta}^{2,0} v-v\right), \partial_{y}^{2} \phi\right)_{\chi^{(\alpha, \beta)}, I}=0, \quad \forall \phi \in \mathcal{P}_{N}^{0,0} .
$$


We now turn to the Legendre rational approximation. Let

$$
\Lambda=\{x \mid 0<x<\infty\} .
$$

The Legendre rational functions of degree $l$ are defined by

$$
R_{l}(x)=\frac{\sqrt{2}}{x+1} L_{l}\left(\frac{x-1}{x+1}\right), \quad l=0,1,2, \cdots .
$$

According to [18], $R_{l}(x)$ are the eigenfunctions of the singular Sturm-Liouville problem

$$
(x+1) \partial_{x}\left(x \partial_{x}((x+1) v(x))\right)+\lambda v(x)=0, \quad x \in \Lambda, l=0,1,2, \cdots,
$$

with the corresponding eigenvalues $\lambda_{l}=l(l+1)$. They satisfy the recurrence relations

$$
R_{l+1}(x)=\frac{2 l+1}{l+1} \frac{x-1}{x+1} R_{l}(x)-\frac{l}{l+1} R_{l-1}(x), \quad l \geq 1,
$$

and

$$
\begin{aligned}
2(2 l+1) R_{l}(x)= & (x+1)^{2}\left(\partial_{x} R_{l+1}(x)-\partial_{x} R_{l-1}(x)\right) \\
& +(x+1)\left(R_{l+1}(x)-R_{l-1}(x)\right) .
\end{aligned}
$$

It can be shown that

$$
\lim _{x \rightarrow \infty}(x+1) R_{l}(x)=\sqrt{2}, \quad \lim _{x \rightarrow \infty} x \partial_{x}\left((x+1) R_{l}(x)\right)=0 .
$$

The set of Legendre rational functions is the complete $L^{2}(\Lambda)$-orthogonal system, i.e.,

$$
\int_{\Lambda} R_{l}(x) R_{m}(x) d x=\left(l+\frac{1}{2}\right)^{-1} \delta_{l, m} .
$$

For any $N \in \mathbb{N}$, we set

$$
\mathcal{R}_{N}=\operatorname{span}\left\{R_{0}, R_{1}, \cdots, R_{N}\right\}, \quad \mathcal{R}_{N}^{0,0}=\mathcal{R}_{N} \cap H_{0}^{2}(\Lambda) .
$$

In order to provide a reasonable algorithm and analyze its convergence properly, we need a specific mapping. To this end, for any $v \in H_{0}^{2}(\Lambda)$, let

$$
v^{*}(y)=\left.\frac{1}{2}(x+1) v(x)\right|_{x=\frac{1+y}{1-y}}=\frac{1}{1-y} v\left(\frac{1+y}{1-y}\right) .
$$


A simple calculation shows $v^{*}(y) \in{ }_{00} H^{2}(I)$. Moveover, (2.10) implies that $R_{l}(x) \rightarrow 0$ and $\partial_{x} R_{l}(x) \rightarrow 0$, as $x \rightarrow \infty$. Therefore, by the definition of ${ }_{00} \tilde{P}_{N, 2,0}^{2}$ and the properties of the Legendre polynomials, we can verify that

$$
\left.(1-y){ }_{00} \tilde{P}_{N, 2,0}^{2} v^{*}(y)\right|_{y=\frac{x-1}{x+1}} \in \mathcal{R}_{N}^{0,0} .
$$

Accordingly, we define the mapping $\Pi_{N}^{2,0}: H_{0}^{2}(\Lambda) \rightarrow \mathcal{R}_{N}^{0,0}$ by

$$
\Pi_{N}^{2,0} v(x)=\left.(1-y){ }_{00} \tilde{P}_{N, 2,0}^{2} v^{*}(y)\right|_{y=\frac{x-1}{x+1}} .
$$

We now introduce the mixed Legendre-Legendre rational approximation. Let $\Omega=\Lambda \times I$ with the boundary $\partial \Omega=\{(x, y) \mid x=0$ or $y= \pm 1\}$. The spaces $H^{r}(\Omega)$ and $H_{0}^{r}(\Omega)$ with the semi-norm $|v|_{H^{r}}$, and norm $\|v\|_{H^{r}}$ have the meanings as usual. In particular, we denote by $(u, v)$ and $\|v\|$ the inner product and norm of $L^{2}(\Omega)$.

For any $v \in H_{0}^{2}(\Omega), v(x, y)=\partial_{x} v(x, y)=\partial_{y} v(x, y)=0$ on $\partial \Omega$. Therefore, we can use the Poincare inequality in one dimension to derive that

$$
\|v\| \leq c\|\nabla v\| \leq c|v|_{H^{2}} .
$$

Moreover, by integration by parts, we assert that for $v \in H_{0}^{2}(\Omega),\|\Delta v\|^{2} \sim|v|_{H^{2}}^{2}$. The previous statements tell us that we may take $(\Delta u, \Delta v)$ as the inner product of $H_{0}^{2}(\Omega)$.

For any

$$
M, N \in \mathbb{N}, V_{N, M}=\mathcal{R}_{N}(\Lambda) \bigotimes \mathcal{P}_{M}(I) \text { and } V_{N, M}^{0,0}=\mathcal{R}_{N}^{0,0}(\Lambda) \bigotimes \mathcal{P}_{M}^{0,0}(I)
$$

The orthogonal projection $P_{N, M}: L^{2}(\Omega) \rightarrow V_{N, M}$ is defined by

$$
\left(P_{N, M} v-v, \phi\right)=0, \quad \forall \phi \in V_{N, M} .
$$

The orthogonal projection $P_{N, M}^{2,0}: H_{0}^{2}(\Omega) \rightarrow V_{N, M}^{0,0}$ is defined by

$$
\left(\Delta\left(P_{N, M}^{2,0} v-v\right), \Delta \phi\right)=0, \quad \forall \phi \in V_{N, M}^{0,0} .
$$

In actual computation and numerical analysis of the mixed spectral method for an infinite strip, we shall also use the mapping $Q_{N, M}^{2,0}: H_{0}^{2}(\Omega) \rightarrow V_{N, M}^{0,0}$, defined by

$$
Q_{N, M}^{2,0} v=\Pi_{N}^{2,0} \tilde{P}_{M, 0,0}^{2,0} v
$$




\subsection{Mixed spectral scheme}

As discussed in Section 1, it is reasonable to use the stream function form of Navier-Stokes equation in numerical simulation of incompressible flow. In order to do this, Guo Ben-yu and coauthors developed the Legendre spectral method for a square and the mixed Legendre-Laguerre spectral method for an infinite strip, see [16, 23, 33]. We now construct the mixed Legendre-Legendre rational spectral method for an infinite strip, which has several advantages in actual computation and theoretical analysis, as described in Section 1.

We denote by $U(x, y, t), U_{0}(x, y), v$ and $F(x, y, t)$ the stream function, the initial state, the kinetic viscosity and the body force, respectively. The stream function form of Navier-Stokes equation is as follows,

$$
\begin{cases}\partial_{t} \Delta U+\partial_{y} U \partial_{x}(\Delta U)-\partial_{x} U \partial_{y}(\Delta U)-v \Delta^{2} U=\nabla \times F, & \text { in } \Omega \times(0, T], \\ U=\frac{\partial U}{\partial n}=0, & \text { on } \partial \Omega \times(0, T], \\ \lim _{x \rightarrow \infty} U=\lim _{x \rightarrow \infty} \partial_{x} U=0, & y \in[-1,1], t \in(0, T], \\ U(x, y, 0)=U_{0}(x, y), & \text { in } \bar{\Omega} .\end{cases}
$$

Let $f=-\nabla \times F$ and the operator $J(u, v, w)=\left(\Delta v, \partial_{y} u \partial_{x} w-\partial_{x} u \partial_{y} w\right)$. The weak formulation of (2.15) is to find $U \in L^{\infty}\left(0, T ; H_{0}^{1}(\Omega)\right) \cap L^{2}\left(0, T ; H_{0}^{2}(\Omega)\right)$ such that

$$
\left\{\begin{array}{l}
\left(\partial_{t} \nabla U, \nabla v\right)+v(\Delta U, \Delta v)+J(U, U, v)=(f, v), \\
\forall v \in H_{0}^{2}(\Omega), t \in(0, T], \\
U(0)=U_{0}
\end{array}\right.
$$

Clearly,

$$
J(u, v, w)+J(w, v, u)=0, \quad J(u, v, u)=0 .
$$

It was shown in [22] that if $U_{0} \in H_{0}^{1}(\Omega)$ and $f \in L^{2}\left(0, T ; H^{-2}(\Omega)\right)$, then (2.16) has a unique solution in $L^{\infty}\left(0, T ; H_{0}^{1}(\Omega)\right) \cap L^{2}\left(0, T ; H_{0}^{2}(\Omega)\right)$.

The mixed Legendre-Legendre rational spectral scheme for (2.16) is to find $u_{N, M}(t) \in V_{N, M}^{0,0}$ such that

$$
\left\{\begin{array}{cl}
\left(\partial_{t} \nabla u_{N, M}(t), \nabla \phi\right)+v\left(\Delta u_{N, M}(t), \Delta \phi\right) & \\
+J\left(u_{N, M}(t), u_{N, M}(t), \phi\right)=(f, \phi), & \forall \phi \in V_{N, M}^{0,0}(\Omega), t \in(0, T], \\
u_{N, M}(0)=u_{0, N, M}, & \text { in } \bar{\Omega} .
\end{array}\right.
$$


We can take $u_{0, N, M}=P_{N, M} U_{0}, P_{N, M}^{2,0} U_{0}$ or $Q_{N, M}^{2,0} U_{0}$.

In the sequel, we denote by $c$ a generic positive constant independent of any function and $N, M$.

By taking $\phi=2 u_{N, M}(t)$ in (2.18) and using (2.17), we obtain that

$$
\begin{aligned}
\frac{d}{d t}\left\|\nabla u_{N, M}(t)\right\|^{2}+2 v\left\|\Delta u_{N, M}(t)\right\|^{2} & \leq 2\|f(t)\|_{H^{-2}}\left\|u_{N, M}(t)\right\|_{H^{2}} \\
& \leq v\left\|\Delta u_{N, M}(t)\right\|^{2}+\frac{c}{v}\|f(t)\|_{H^{-2}}^{2} .
\end{aligned}
$$

Hence,

$$
\begin{gathered}
\left\|u_{N, M}\right\|_{L^{\infty}\left(0, T ; H^{1}(\Omega)\right)}^{2}+v\left\|u_{N, M}\right\|_{L^{2}\left(0, T ; H^{2}(\Omega)\right)}^{2} \\
\leq c\left\|\nabla u_{0, N, M}\right\|^{2}+\frac{c}{v}\|f\|_{L^{2}\left(0, T ; H^{-2}(\Omega)\right)}^{2} .
\end{gathered}
$$

\subsection{The stability and convergence}

In this subsection, we state the results on the stability and convergence of scheme (2.18), which will be proved in section 3 .

Theorem 2.1. Assume that $u_{0, N, M}$ and $f$ have the errors $\tilde{u}_{0, N, M}$ and $\tilde{f}$, respectively, which induce the error of $u_{N, M}$, denoted by $\tilde{u}_{N, M}$. Then there exists a positive constant $d^{*}$ depending only on $\left\|u_{N, M}\right\|_{L^{2}\left(0, T ; H^{2}(\Omega)\right)}$, v and $T$ such that

$$
\begin{aligned}
& \left\|\tilde{u}_{N, M}\right\|_{L^{\infty}\left(0, T ; H^{1}(\Omega)\right)}+\left\|\tilde{u}_{N, M}\right\|_{L^{2}\left(0, T ; H^{2}(\Omega)\right)} \\
& \leq d^{*}\left(\left\|\tilde{u}_{0, N, M}\right\|_{H^{1}(\Omega)}+\|\tilde{f}\|_{L^{2}\left(0, T ; L^{2}(\Omega)\right)}\right) .
\end{aligned}
$$

In order to describe the numerical accuracy properly, we introduce the space $H_{A}^{r}(\Lambda)$. For any integer $r \geq 0$, its norm is given by

$$
\|v\|_{r, A, \Lambda}=\left(\sum_{k=0}^{r} \int_{\Lambda}\left(\partial_{x}^{k} v(x)\right)^{2}(x+1)^{2 k+r} d x\right)^{\frac{1}{2}} .
$$

For any $r>0$, we define the space $H_{A}^{r}(\Lambda)$ by space interpolation. We also introduce the non-isotropic space

$$
H^{r, s}(\Omega)=L^{2}\left(I, H^{r}(\Lambda)\right) \cap H^{s}\left(I, L^{2}(\Lambda)\right), \quad r, s \geq 0,
$$


equipped with the norms

$$
\|v\|_{H^{r, s}(\Omega)}=\left(\|v\|_{L^{2}\left(I, H^{r}(\Lambda)\right)}^{2}+\|v\|_{H^{s}\left(I, L^{2}(\Lambda)\right)}^{2}\right)^{\frac{1}{2}} .
$$

Furthermore, we define the space $M^{r, s}(\Omega)$. For any integer $r, s \geq 2$, its norm is given by

$$
=\left(\sum_{k=0}^{1}\left(\int_{\Lambda} d x \int_{I}\left(\partial_{y}^{s-2 k} \partial_{M^{r, s}(\Omega)}^{2 k} v\right)^{2}\left(1-y^{2}\right)^{s-2 k-2} d y+\left\|\partial_{y}^{2 k} v\right\|_{L^{2}\left(I, H_{A}^{r-2 k}(\Lambda)\right)}^{2}\right)\right)^{\frac{1}{2}} .
$$

For any $r, s>2$, we define the space $M^{r, s}(\Omega)$ by space interpolation. Especially,

$$
H^{r}(\Omega)=H^{r, r}(\Omega) \quad \text { and } \quad H_{0}^{r}(\Omega)=L^{2}\left(I, H_{0}^{r}(\Lambda)\right) \cap H_{0}^{r}\left(I, L^{2}(\Lambda)\right) .
$$

For the sake of simplicity, we also denote the norms $\|\cdot\|_{H^{r, s}(\Omega)}$ and $\|\cdot\|_{M^{r, s}(\Omega)}$ by $\|\cdot\|_{H^{r, s}}$ and $\|\cdot\|_{M^{r, s}}$, respectively.

Theorem 2.2. Let $U$ and $u_{N, M}$ be the solutions of (2.16) and (2.18), respectively. If for integers $r, s \geq 2, U \in H^{1}\left(0, T ; M^{r, s}(\Omega)\right)$, then for $0 \leq t \leq T$,

$$
\left\|U-u_{N, M}\right\|_{L^{\infty}\left(0, T ; H^{1}(\Omega)\right)}+\left\|U-u_{N, M}\right\|_{L^{2}\left(0, T ; H^{2}(\Omega)\right)} \leq d_{*}\left(N^{2-r}+M^{2-s}\right),
$$

where $d_{*}$ is a positive constant depending only on $v, \Omega, T$ and the norms of $U$ in the mentioned spaces.

\subsection{Numerical results}

We first choose the base functions of $V_{N, M}^{0,0}$ suitably. As in [28], let

$$
\phi_{j}(x)=R_{j}(x)-\frac{2(2 j+5)}{2 j+7} R_{j+2}(x)+\frac{2 j+3}{2 j+7} R_{j+4}(x), \quad 0 \leq j \leq N-4,
$$

and

$$
\begin{gathered}
\psi_{k}(y)=d_{k}\left(L_{k}(y)-\frac{2(2 k+5)}{2 k+7} L_{k+2}(y)+\frac{2 k+3}{2 k+7} L_{k+4}(y)\right) \\
d_{k}=\frac{1}{\sqrt{2(2 k+3)^{2}(2 k+5)}}, \quad 0 \leq k \leq M-4
\end{gathered}
$$


Obviously,

$$
\mathcal{R}_{N}^{0,0}=\left\{\phi_{j}, 0 \leq j \leq N-4\right\}, \quad \mathcal{P}_{M}^{0,0}=\left\{\psi_{k}, 0 \leq k \leq M-4\right\} .
$$

Therefore, we take the base functions of $V_{N, M}^{0,0}$ as

$$
\Psi_{j, k}=\phi_{j}(x) \psi_{k}(y), \quad 0 \leq j \leq N-4,0 \leq k \leq M-4 .
$$

The numerical solution is expanded as

$$
u_{N, M}(x, y, t)=\sum_{j=0}^{N-4} \sum_{k=0}^{M-4} \hat{u}_{N, M}(t) \phi_{j}(x) \psi_{k}(y) .
$$

Inserting the above expression into (2.18), we obtain a system of ordinary differential equation with unknown function $\hat{u}_{N, M}(t)$. For temporal discretization, we use the standard Runge-Kutta method of fourth-order, with step size $\tau$.

Next, for description of numerical errors, let $\zeta_{N, j}$ and $\sigma_{M, k}$ the distinct roots of $R_{N+1}(x)$ and $L_{M+1}(y)$, respectively. The corresponding weights are denoted by $\omega_{N, j}$ and $\rho_{M, k}$, see $[2,18]$. The error

$$
E_{N, M}(t)=\left(\sum_{j=0}^{N} \sum_{k=0}^{M}\left(U\left(\zeta_{N, j}, \sigma_{M, k}, t\right)-u_{N, M}\left(\zeta_{N, j}, \sigma_{M, k}, t\right)\right)^{2} \omega_{N, j} \rho_{M, k}\right)^{\frac{1}{2}}
$$

Take the test function

$$
U(x, y, t)=\frac{x^{2}\left(1-y^{2}\right)^{2} \sin (k t)}{(2+x+y)^{h}} .
$$

We use (2.18) with $u_{0, N, M}=P_{N, M}^{2,0} U_{0}$ to solve (2.16) numerically.

In Table 1, we present the error $E_{N, M}(t)$ at $t=1$ for various values of $N, M$ and $\tau$. Clearly, the proposed scheme (2.18) provides very accurate numerical solution even for small $v$ and moderate values of $N, M$ and $\tau$. They also demonstrate that the error decays fast as $N$ and $M$ increase and $\tau$ decreases. This coincides well with theoretical analysis.

In Table 2, we present the error $E_{N, M}(t)$ at various values of $t$. Clearly, the calculation is quite stable. 


\begin{tabular}{cccc}
\hline$\tau$ & $\mathrm{N}=12, \mathrm{M}=12$ & $\mathrm{~N}=16, \mathrm{M}=16$ & $\mathrm{~N}=24, \mathrm{M}=16$ \\
\hline 0.01 & $6.780 \mathrm{e}-07$ & $2.098 \mathrm{e}-08$ & $2.371 \mathrm{e}-09$ \\
\hline 0.005 & $6.779 \mathrm{e}-07$ & $2.097 \mathrm{e}-08$ & $1.276 \mathrm{e}-09$ \\
\hline 0.001 & $6.778 \mathrm{e}-07$ & $2.096 \mathrm{e}-08$ & $1.212 \mathrm{e}-09$ \\
\hline
\end{tabular}

Table 1 - The errors $E_{N, M}(1), v=10^{-3}, k=0.2$ and $h=6$.

\begin{tabular}{cc}
\hline $\mathrm{t}$ & $E_{N, M}(t)$ \\
\hline 1 & $2.098 \mathrm{e}-08$ \\
\hline 2 & $4.258 \mathrm{e}-08$ \\
\hline 3 & $7.919 \mathrm{e}-08$ \\
\hline 4 & $1.780 \mathrm{e}-07$ \\
\hline
\end{tabular}

Table 2 - The errors $E_{N, M}(t), v=10^{-3}, k=0.2$, $h=6, N=M=16$ and $\tau=10^{-2}$.

Table 3 is for the error $E_{N, M}(t)$ at $t=1$ with various values of $h$. It indicates that the errors decay fast as $h$ increases. In fact, the exact solution is smoother for larger $h$. Therefore, as predicted by Theorem 2.2, the numerical result is more accurate for smoother solution.

In Table 4, we present the error $E_{N, M}(t)$ at $t=1$ for various values of $\nu$. We find that scheme (2.18) is very efficient even for very small $v$.

To compare our results with the results in [23], we take the test function

$$
U(x, y, t)=\frac{x^{2}\left(1-y^{2}\right)^{2} \sin (k x t+k y t)}{(2+x+y)^{h}} e^{-\frac{x}{2}},
$$

which corresponds to the function $W(x, y, t)$ in (3.1) of [23]. In Table 5, we present the error $E_{N, M}(t)$ of scheme (2.18) at $t=1$ and the corresponding results

\begin{tabular}{cc}
\hline $\mathrm{h}$ & $E_{N, M}(t)$ \\
\hline 4 & $2.945 \mathrm{e}-04$ \\
\hline 5 & $2.831 \mathrm{e}-06$ \\
\hline 6 & $1.091 \mathrm{e}-06$ \\
\hline 7 & $2.043 \mathrm{e}-07$ \\
\hline
\end{tabular}

Table 3 - The errors $E_{N, M}(t), v=10^{-2}, k=0.2$, $h=6, N=M=12$ and $\tau=10^{-2}$. 


\begin{tabular}{cccc}
\hline$v$ & $\mathrm{~N}=8, \mathrm{M}=8$ & $\mathrm{~N}=10, \mathrm{M}=10$ & $\mathrm{~N}=12, \mathrm{M}=12$ \\
\hline $10^{-2}$ & $1.159005 \mathrm{e}-05$ & $2.313422 \mathrm{e}-06$ & $1.091073 \mathrm{e}-06$ \\
\hline $10^{-3}$ & $1.156591 \mathrm{e}-05$ & $2.212567 \mathrm{e}-06$ & $6.780100 \mathrm{e}-07$ \\
\hline $10^{-4}$ & $1.156556 \mathrm{e}-05$ & $2.212266 \mathrm{e}-06$ & $6.777824 \mathrm{e}-07$ \\
\hline $10^{-5}$ & $1.156553 \mathrm{e}-05$ & $2.212258 \mathrm{e}-06$ & $6.777821 \mathrm{e}-07$ \\
\hline
\end{tabular}

Table 4 - The errors $E_{N, M}(1), k=0.2, h=6$ and $\tau=10^{-2}$.

\begin{tabular}{c|cccc}
\hline & $\mathrm{N}=8, \mathrm{M}=8$ & $\mathrm{~N}=20, \mathrm{M}=12$ & $\mathrm{~N}=40, \mathrm{M}=16$ & $\mathrm{~N}=68, \mathrm{M}=20$ \\
\hline Scheme (2.18) & $8.047 \mathrm{e}-03$ & $4.662 \mathrm{e}-04$ & $3.138 \mathrm{e}-06$ & $4.142 \mathrm{e}-07$ \\
\hline Scheme in [23] & $1.652 \mathrm{e}-03$ & $1.056 \mathrm{e}-04$ & $2.745 \mathrm{e}-05$ & $2.517 \mathrm{e}-06$ \\
\hline
\end{tabular}

Table 5 - The errors $E_{N, M}(1), v=10^{-5}, k=0.2, h=4$ and $\tau=10^{-2}$.

in Table 1 of [23]. Clearly, the proposed scheme (2.18) provides more accurate numerical results for larger values of $N, M$.

It is noted that the values of $N$ and $M$ in Table 5 correspond to the values of $N+4$ and $M+4$ in Table 1 of [23], respectively.

\section{Analysis of stability and convergence}

In this section, we first establish some basic results on the mixed LegendreLegendre rational approximation which form the mathematical foundation of the related spectral methods for various differential equations in an infinite strip. Then we use these results to prove the stability and convergence of scheme (2.18), stated in Theorems 2.1 and 2.2.

\subsection{Some approximation results}

Let $\alpha, \beta, \gamma, \delta, \sigma, \lambda>-1$, and introduce the space $H_{\alpha, \beta, \gamma, \delta, \sigma, \lambda}^{\mu}(I), 0 \leq \mu \leq 2$. For $\mu=0, H_{\alpha, \beta, \gamma, \delta, \sigma, \lambda}^{0}(I)=L_{\chi^{(\sigma, \lambda)}}^{2}(I)$. For $\mu=2$,

$$
H_{\alpha, \beta, \gamma, \delta, \sigma, \lambda}^{2}(I)=\left\{v \mid v \text { is measurable and }\|v\|_{2, \alpha, \beta, \gamma, \delta, \sigma, \lambda}<\infty\right\}
$$

with the norm

$$
\|v\|_{2, \alpha, \beta, \gamma, \delta, \sigma, \lambda, I}=\left(|v|_{2, \chi^{(\alpha, \beta), I}}^{2}+|v|_{1, \chi^{(\gamma, \delta), I}}^{2}+\|v\|_{\chi^{(\sigma, \lambda), I}}^{2}\right)^{\frac{1}{2}} .
$$


For $0<\mu<2$, the space $H_{\alpha, \beta, \gamma, \delta, \sigma, \lambda}^{\mu}(I)$ is defined by the space interpolation as in [1]. Its norm is denoted by $\|v\|_{\mu, \alpha, \beta, \gamma, \delta, \sigma, \lambda, I}$.

For description of approximation results, we also define the space $H_{\chi^{(\alpha, \beta)}, *}^{r}(I)$. For any integer $r \geq 2$, its norm and semi-norm are given by

$$
\begin{aligned}
\|v\|_{r, \chi}^{(\alpha, \beta)}, *, I & =\left(\sum_{k=0}^{r-2}\left\|\partial_{y}^{k+2} v\right\|_{\chi^{(\alpha+k, \beta+k), I}}^{2}\right)^{\frac{1}{2}}, \\
|v|_{r, \chi^{(\alpha, \beta)}, *, I} & =\left\|\partial_{y}^{r} v\right\|_{\chi^{(\alpha+r-2, \beta+r-2), I} .}
\end{aligned}
$$

For any $r>2$, we define the space $H_{\chi^{(\alpha, \beta)}, *}^{r}(I)$ by space interpolation.

Lemma 3.1. If $\alpha \leq \min (\gamma+2, \sigma+4), \beta \leq 0$ and $\delta, \lambda \geq 0$, then for any $v \in{ }_{00} H_{\chi^{(\alpha, \beta)}}^{2}(I) \cap H_{\chi^{(\alpha, \beta)}, *}^{r}(I), r \in \mathbb{N}$ and $r \geq 2$,

$$
\left\|{ }_{00} \tilde{P}_{N, \alpha, \beta}^{2} v-v\right\|_{2, \alpha, \beta, \gamma, \delta, \sigma, \lambda, I} \leq c N^{2-r}|v|_{r, \chi^{(\alpha, \beta)}, *, I} .
$$

If, in addition, $\alpha \leq \sigma+2$ and $\beta \leq \lambda+2$, then for $0 \leq \mu \leq 2$,

$$
\left\|{ }_{00} \tilde{P}_{N, \alpha, \beta}^{2} v-v\right\|_{\mu, \alpha, \beta, \gamma, \delta, \sigma, \lambda, I} \leq c N^{\mu-r}|v|_{r, \chi(\alpha, \beta), *, I} .
$$

Lemma 3.2. If $-1<\alpha, \beta<1, \alpha \leq \gamma+2$ and $\beta \leq \delta+2$, then for any $v \in H_{0, \chi^{(\alpha, \beta)}}^{2}(I) \cap H_{\chi^{(\alpha, \beta)}, *}^{r}(I), r \in \mathbb{N}$ and $r \geq 2$,

$$
\left\|\tilde{P}_{N, \alpha, \beta}^{2,0} v-v\right\|_{2, \alpha, \beta, \gamma, \delta, \sigma, \lambda, I} \leq c N^{2-r}|v|_{r, \chi^{(\alpha, \beta)}, *, I} .
$$

In particular, for $\alpha=\beta=0$ and $0 \leq \mu \leq 2$,

$$
\left\|\tilde{P}_{N, \alpha, \beta}^{2,0} v-v\right\|_{\mu, \alpha, \beta, \gamma, \delta, \sigma, \lambda, I} \leq c N^{\mu-r}|v|_{r, \chi^{(\alpha, \beta), *, I}} .
$$

Lemmas 3.1 and 3.2 come from Theorems 2.3 and 2.5 of [21], respectively.

Lemma 3.3. For any $v \in H_{0}^{2}(\Lambda) \cap H_{A}^{r}(\Lambda)$ and $0 \leq \mu \leq 2 \leq r$,

$$
\left\|\Pi_{N}^{2,0} v-v\right\|_{\mu, \Lambda} \leq c N^{\mu-r}\|v\|_{r, A, \Lambda} .
$$


Proof. By using (2.12), (2.13) and (3.2), a direct calculation leads to that

$$
\begin{aligned}
\left\|\Pi_{N}^{2,0} v-v\right\|_{L^{2}(\Lambda)} & =\sqrt{2}\left(\int_{I}\left({ }_{00} \tilde{P}_{N, 2,0}^{2} v^{*}(y)-v^{*}(y)\right)^{2} d y\right)^{\frac{1}{2}} \\
& \leq c N^{-r}\left|v^{*}\right|_{H_{\chi(2,0), *}^{r}} .
\end{aligned}
$$

Moreover, we use induction to show that for $k \in \mathbb{N}$,

$$
\partial_{y}^{k} v^{*}(y)=\left.\sum_{j=0}^{k} c_{j}(1+x)^{k+j+1} \partial_{x}^{j} v(x)\right|_{x=\frac{1+y}{1-y}},
$$

$c_{j}$ being certain positive constants. Hence, $\left|v^{*}\right|_{H_{\chi^{(2,0), *}}^{r}(I)} \leq c\|v\|_{r, A, \Lambda}$, and so $\left\|\Pi_{N}^{2,0} v-v\right\|_{L^{2}(\Lambda)} \leq c N^{-r}\|v\|_{r, A, \Lambda}$. For $\mu=2$, we use (2.13) and (3.1) to deduce that

$$
\begin{aligned}
\left\|\Pi_{N}^{2,0} v-v\right\|_{2, \Lambda} \leq & c\left(\int_{I}\left({ }_{00} \tilde{P}_{N, 2,0}^{2} v^{*}(y)-v^{*}(y)\right)^{2} d y\right. \\
& +\int_{I}\left(\partial_{y}\left({ }_{00} \tilde{P}_{N, 2,0}^{2} v^{*}(y)-v^{*}(y)\right)\right)^{2}(1-y)^{4} d y \\
& \left.+\int_{I}\left(\partial_{y}^{2}\left({ }_{00} \tilde{P}_{N, 2,0}^{2} v^{*}(y)-v^{*}(y)\right)\right)^{2}(1-y)^{8} d y\right)^{\frac{1}{2}} \\
\leq & c\left(\int_{I}\left({ }_{00} \tilde{P}_{N, 2,0}^{2} v^{*}(y)-v^{*}(y)\right)^{2} d y\right. \\
& +\int_{I}\left(\partial_{y}\left({ }_{00} \tilde{P}_{N, 2,0}^{2} v^{*}(y)-v^{*}(y)\right)\right)^{2}(1-y)^{4} d y \\
& \left.+\int_{I}\left(\partial_{y}^{2}\left({ }_{00} \tilde{P}_{N, 2,0}^{2} v^{*}(y)-v^{*}(y)\right)\right)^{2}(1-y)^{2} d y\right)^{\frac{1}{2}} \\
\leq & c N^{2-r}\left|v^{*}\right|_{H_{\chi}{ }^{(2,0), *}{ }^{(I)}} \leq c N^{2-r}\|v\|_{r, A, \Lambda} .
\end{aligned}
$$

The result with $0<\mu<2$ follows from the previous statements and space interpolation.

We now state the main approximation results of this section.

Lemma 3.4. For any $v \in M^{r, s}(\Omega) \cap H_{0}^{2}(\Omega)$ and integers $r, s \geq 2$,

$$
\left\|v-Q_{N, M}^{2,0} v\right\|_{H^{2}(\Omega)} \leq c\left(N^{2-r}+M^{2-s}\right)\|v\|_{M^{r, s}} .
$$


Proof. For simplicity of statements, we use the notations

$$
\begin{aligned}
B_{1} & =\left\|\partial_{x}^{2}\left(v-\Pi_{N}^{2,0} \tilde{P}_{M, 0,0}^{2,0} v\right)\right\|, \\
B_{2} & =\left\|\partial_{y}^{2}\left(v-\Pi_{N}^{2,0} \tilde{P}_{M, 0,0}^{2,0} v\right)\right\|, \\
B_{3} & =\left\|\partial_{x} \partial_{y}\left(v-\Pi_{N}^{2,0} \tilde{P}_{M, 0,0}^{2,0} v\right)\right\| .
\end{aligned}
$$

By virtue of (3.4) and (3.5), we deduce that

$$
\begin{aligned}
B_{1} \leq & \left\|\partial_{x}^{2}\left(v-\Pi_{N}^{2,0} v\right)\right\|+\left\|\partial_{x}^{2} \Pi_{N}^{2,0} v-\tilde{P}_{M, 0,0}^{2,0}\left(\partial_{x}^{2} \Pi_{N}^{2,0} v\right)\right\| \\
\leq & c N^{2-r}\|v\|_{L^{2}\left(I, H_{A}^{r}(\Lambda)\right)} \\
& \quad+c M^{2-s}\left(\int_{\Lambda} d x \int_{I}\left(\partial_{y}^{s-2}\left(\partial_{x}^{2} \Pi_{N}^{2,0} v\right)\right)^{2}\left(1-y^{2}\right)^{s-4} d y\right)^{\frac{1}{2}} \\
& \leq c\left(M^{2-s}+N^{2-r}\right)\|v\|_{M^{r, s}}
\end{aligned}
$$

Similarly,

$$
\begin{aligned}
B_{2} \leq & \left\|\partial_{y}^{2}\left(v-\tilde{P}_{M, 0,0}^{2,0} v\right)\right\|+\left\|\partial_{y}^{2} \tilde{P}_{M, 0,0}^{2,0} v-\Pi_{N}^{2,0}\left(\partial_{y}^{2} \tilde{P}_{M, 0,0}^{2,0} v\right)\right\| \\
\leq & c M^{2-s}\left(\int_{\Lambda} d x \int_{I}\left(\partial_{y}^{s} v\right)^{2}\left(1-y^{2}\right)^{s-2} d y\right)^{\frac{1}{2}} \\
& \quad+c N^{2-r}\left\|\partial_{y}^{2} \tilde{P}_{M, 0,0}^{2,0} v\right\|_{L^{2}\left(I, H_{A}^{r-2}(\Lambda)\right)} \\
\leq & c\left(M^{2-s}+N^{2-r}\right)\|v\|_{M^{r, s}} .
\end{aligned}
$$

By integration by parts,

$$
\begin{aligned}
B_{3} & =\left(\int_{\Lambda} \int_{I} \partial_{x}^{2}\left(v-\Pi_{N}^{2,0} \tilde{P}_{M, 0,0}^{2,0} v\right) \partial_{y}^{2}\left(v-\Pi_{N}^{2,0} \tilde{P}_{M, 0,0}^{2,0} v\right) d y d x\right)^{\frac{1}{2}} \\
& \leq \frac{1}{2}\left(B_{1}+B_{2}\right) .
\end{aligned}
$$

Thus, by using (3.7)-(3.9) and the Poincare inequality (2.14), we obtain the desired result (3.6). 
Lemma 3.5. For any $v \in M^{r, s}(\Omega) \cap H_{0}^{2}(\Omega)$ and integers $r, s \geq 2$,

$$
\left\|v-P_{N, M}^{2,0} v\right\|_{H^{2}(\Omega)} \leq c\left(N^{2-r}+M^{2-s}\right)\|v\|_{M^{r, s}} .
$$

Proof. By projection theorem,

$$
\left\|\Delta\left(v-P_{N, M}^{2,0} v\right)\right\| \leq\|\Delta(v-\phi)\|, \quad \forall \phi \in V_{N, M}^{0,0} .
$$

Taking $\phi=Q_{N, M}^{2,0} v$, we have from (3.6) and the Poincare inequality (2.14) that

$$
\begin{aligned}
\left\|v-P_{N, M}^{2,0} v\right\|_{H^{2}(\Omega)} & \leq c\left\|\Delta\left(v-P_{N, M}^{2,0} v\right)\right\| \\
& \leq c\left\|\Delta\left(v-Q_{N, M}^{2,0} v\right)\right\| \\
& \leq c\left(N^{2-r}+M^{2-s}\right)\|v\|_{M^{r, s}} .
\end{aligned}
$$

\subsection{Proofs of stability and convergence}

We are now in position of proving Theorems 2.1 and 2.2. We shall use two embedding inequalities. In fact, for any $u, v, w \in H_{0}^{2}(\Omega)$ (see [22]),

$$
\begin{gathered}
|J(u, v, w)| \leq 2\|\Delta u\|\|\Delta v\|\|\Delta w\|, \\
|J(u, u, v)|=|J(v, u, u)| \leq 2\|\nabla u\|\|\Delta u\|\|\Delta v\| .
\end{gathered}
$$

We first prove Theorem 2.1. According to (2.18), the error $\tilde{u}_{N, M}$ satisfies the following equation,

$$
\begin{array}{r}
\left(\partial_{t} \nabla \tilde{u}_{N, M}(t), \nabla \phi\right)+v\left(\Delta \tilde{u}_{N, M}(t), \Delta \phi\right)+J\left(u_{N, M}(t), \tilde{u}_{N, M}(t), \phi\right) \\
+J\left(\tilde{u}_{N, M}(t), u_{N, M}(t), \phi\right)+J\left(\tilde{u}_{N, M}(t), \tilde{u}_{N, M}(t), \phi\right)=(\tilde{f}, \phi) .
\end{array}
$$

Take $\phi=2 \tilde{u}_{N, M}$ in (3.13). It follows from (2.17) that

$$
\frac{d}{d t}\left\|\nabla \tilde{u}_{N, M}\right\|^{2}+2 v\left\|\Delta \tilde{u}_{N, M}\right\|^{2}+2 J\left(u_{N, M}, \tilde{u}_{N, M}, \tilde{u}_{N, M}\right)=2\left(\tilde{f}, \tilde{u}_{N, M}\right) .
$$

Thanks to (2.17), (3.12) and the Cauchy inequality,

$$
\begin{aligned}
\left|J\left(u_{N, M}, \tilde{u}_{N, M}, \tilde{u}_{N, M}\right)\right| & =\left|J\left(\tilde{u}_{N, M}, \tilde{u}_{N, M}, u_{N, M}\right)\right| \\
& \leq 2\left\|\nabla \tilde{u}_{N, M}\right\|\left\|\Delta \tilde{u}_{N, M}\right\|\left\|\Delta u_{N, M}\right\| \\
& \leq \frac{v}{2}\left\|\Delta \tilde{u}_{N, M}\right\|^{2}+\frac{2}{v}\left\|\nabla \tilde{u}_{N, M}\right\|^{2}\left\|\Delta u_{N, M}\right\|^{2}
\end{aligned}
$$


By the Poincare inequality (2.14),

$$
2\left|\left(\tilde{f}, \tilde{u}_{N, M}\right)\right| \leq 2\|\tilde{f}\|\left\|\tilde{u}_{N, M}\right\| \leq\left\|\nabla \tilde{u}_{N, M}\right\|^{2}+c\|\tilde{f}\|^{2} .
$$

Consequently, (3.14) reads

$$
\begin{aligned}
\frac{d}{d t}\left\|\nabla \tilde{u}_{N, M}\right\|^{2} & +v\left\|\Delta \tilde{u}_{N, M}\right\|^{2} \leq\left(1+\frac{4}{v}\left\|\Delta u_{N, M}\right\|^{2}\right)\left\|\nabla \tilde{u}_{N, M}\right\|^{2} \\
& +c\|\tilde{f}\|^{2} .
\end{aligned}
$$

Finally, integrating (3.15) with respect to $t$ and using the Gronwall inequality, we reach the desired result in Theorem 2.1.

We next prove Theorem 2.2. For simplicity, we focus on the case $u_{0, N, M}=$ $P_{N, M}^{2,0} U_{0}$. Let $U_{N, M}=P_{N, M}^{2,0} U$. We have from (2.16) that

$$
\begin{aligned}
\left(\partial_{t} \nabla U_{N, M}(t), \nabla \phi\right) & +v\left(\Delta U_{N, M}(t), \Delta \phi\right)+J\left(U_{N, M}(t), U_{N, M}(t), \phi\right) \\
& +\sum_{j=1}^{3} G_{j}(\phi, t)=(f(t), \phi)
\end{aligned}
$$

where

$$
\begin{aligned}
& G_{1}(\phi, t)=\left(\partial_{t} \nabla\left(U(t)-U_{N, M}(t)\right), \nabla \phi\right), \\
& G_{2}(\phi, t)=J\left(U(t)-U_{N, M}(t), U_{N, M}(t), \phi\right), \\
& G_{3}(\phi, t)=J\left(U(t), U(t)-U_{N, M}(t), \phi\right) .
\end{aligned}
$$

Further, let $\tilde{U}_{N, M}=u_{N, M}-U_{N, M}$. Then subtracting (3.16) from (2.18) yields that

$$
\begin{aligned}
& \left(\partial_{t} \nabla \tilde{U}_{N, M}(t), \nabla \phi\right)+v\left(\Delta \tilde{U}_{N, M}(t), \Delta \phi\right) \\
& \quad+J\left(\tilde{U}_{N, M}(t), U_{N, M}(t)+\tilde{U}_{N, M}(t), \phi\right)=\sum_{j=1}^{4} G_{j}(\phi, t),
\end{aligned}
$$

where $G_{4}(\phi, t)=-J\left(U_{N, M}(t), \tilde{U}_{N, M}(t), \phi\right)$. In addition, $\tilde{U}_{N, M}(0)=0$. Take $\phi=2 \tilde{U}_{N, M}$ in (3.17). Then we use (2.17) to deduce that

$$
\frac{d}{d t}\left\|\nabla \tilde{U}_{N, M}(t)\right\|^{2}+2 v\left\|\Delta \tilde{U}_{N, M}(t)\right\|^{2}=2 \sum_{j=1}^{4} G_{j}\left(\tilde{U}_{N, M}, t\right) .
$$


Next, we estimate $\left|G_{j}\left(\tilde{U}_{N, M}, t\right)\right|, 1 \leq j \leq 4$. By the Cauchy inequality and integration by parts, we deduce that

$$
\begin{aligned}
\left|G_{1}\left(\tilde{U}_{N, M}, t\right)\right| & =\left(\partial_{t}\left(U(t)-U_{N, M}(t)\right), \Delta \tilde{U}_{N, M}(t)\right) \\
& \leq \frac{v}{8}\left\|\Delta \tilde{U}_{N, M}(t)\right\|^{2}+\frac{c}{v}\left\|\partial_{t}\left(U(t)-U_{N, M}(t)\right)\right\|^{2} .
\end{aligned}
$$

By using (3.11) and the Cauchy inequality,

$$
\begin{aligned}
\left|G_{2}\left(\tilde{U}_{N, M}, t\right)\right| & \leq 2\left\|\Delta\left(U(t)-U_{N, M}(t)\right)\right\|\left\|\Delta U_{N, M}(t)\right\|\left\|\Delta \tilde{U}_{N, M}(t)\right\| \\
& \leq \frac{v}{8}\left\|\Delta \tilde{U}_{N, M}(t)\right\|^{2} \\
& +\frac{c}{v}\left\|\Delta U_{N, M}(t)\right\|^{2}\left\|\Delta\left(U(t)-U_{N, M}(t)\right)\right\|^{2} .
\end{aligned}
$$

Similarly,

$$
\begin{aligned}
\left|G_{3}\left(\tilde{U}_{N, M}, t\right)\right| & \leq 2\|\Delta U\|\left\|\Delta\left(U(t)-U_{N, M}(t)\right)\right\|\left\|\Delta \tilde{U}_{N, M}(t)\right\| \\
& \leq \frac{v}{8}\left\|\Delta \tilde{U}_{N, M}(t)\right\|^{2} \\
& +\frac{c}{v}\|\Delta U(t)\|^{2}\left\|\Delta\left(U(t)-U_{N, M}(t)\right)\right\|^{2} .
\end{aligned}
$$

Also, using (3.12) gives that

$$
\begin{aligned}
\left|G_{4}\left(\tilde{U}_{N, M}, t\right)\right| & \leq 2\left\|\Delta \tilde{U}_{N, M}(t)\right\|\left\|\Delta U_{N, M}(t)\right\|\left\|\nabla \tilde{U}_{N, M}(t)\right\| \\
& \leq \frac{v}{8}\left\|\Delta \tilde{U}_{N, M}(t)\right\|^{2} \\
& +\frac{c}{v}\left\|\Delta U_{N, M}(t)\right\|^{2}\left\|\nabla \tilde{U}_{N, M}(t)\right\|^{2} .
\end{aligned}
$$

Furthermore, due to (3.10), we assert that

$$
\begin{aligned}
& \left\|\Delta U_{N, M}(t)\right\| \leq\|\Delta U(t)\|+\left\|\Delta\left(U(t)-U_{N, M}(t)\right)\right\| \leq c\|U(t)\|_{M^{2,2}}, \\
& \left\|\Delta\left(U(t)-U_{N, M}(t)\right)\right\| \leq c\left(N^{2-r}+M^{2-s}\right)\|U(t)\|_{M^{r, s},} \\
& \left\|\partial_{t}\left(U(t)-U_{N, M}(t)\right)\right\| \leq c\left(N^{2-r}+M^{2-s}\right)\left\|\partial_{t} U(t)\right\|_{M^{r, s}, \quad r, s \geq 2 .}
\end{aligned}
$$

Substituting (3.19)-(3.25) into (3.18), we obtain that

$$
\begin{aligned}
\frac{d}{d t}\left\|\nabla \tilde{U}_{N, M}(t)\right\|^{2} & +v\left\|\Delta \tilde{U}_{N, M}(t)\right\|^{2} \leq \frac{c}{v}\|U(t)\|_{M^{2,2}}^{2}\left\|\nabla \tilde{U}_{N, M}(t)\right\|^{2} \\
& +V(t),
\end{aligned}
$$


where

$$
V(t)=\frac{c}{v}\left(N^{2-r}+M^{2-s}\right)^{2}\left(\left\|\partial_{t} U(t)\right\|_{M^{r, s}}^{2}+\|U(t)\|_{M^{2,2}}^{2}\|U(t)\|_{M^{r, s}}^{2}\right) .
$$

Integrating the above with respect to $t$ and using the Gronwall inequality, we obtain that

$$
\left\|\nabla \tilde{U}_{N, M}(t)\right\|^{2}+v \int_{0}^{t}\left\|\Delta \tilde{U}_{N, M}(\xi)\right\|^{2} d \xi \leq e^{\frac{c}{\nu}\|U\|_{L^{2}\left(0, T ; M^{2,2}(\Omega)\right)}^{2}} \int_{0}^{t} V(\xi) d \xi .
$$

This leads to the desired result in Theorem 2.2.

Remark 3.1. If we take $u_{0, N, M}=P_{N, M} U_{0}$ or $Q_{N, M}^{2,0} U_{0}$, then we can follow the same line of proof as in the above to obtain similar results.

\section{Concluding remarks}

In this paper, we consider numerical simulation of the Navier-Stokes equation which plays an important role in studying incompressible viscous fluid flow. Since we use a numerical algorithm based on the stream function form, the numerical solution fulfills the incompressibility automatically. Moreover, it keeps the physical boundary condition on the stream function. Therefore, we do not need to construct free-divergence base functions. Indeed, this is not an easy job for spectral methods. Additionally, we have avoided non-physical boundary conditions on the pressure or the vorticity, which usually creates numerical boundary layers.

In this paper, we use the mixed Legendre-Legendre rational spectral method for the incompressible fluid flow in an infinite strip. The main characters are the followings:

- We approximate the stream function form directly. This fact simplifies actual computation and numerical analysis. It is also easier to generalize the proposed method to multi-dimensional problems.

- We take the Legendre rational functions as the base functions, which are mutually orthogonal with the same weight function as in the continuous 
version. Thus, the corresponding numerical solution keeps the same conservation as the exact solution. This feature leads to more appropriate numerical results, and simplifies computation and theoretical analysis.

- Since the base functions are derived from the Legendre polynomials, we can use the existing code for the Legendre approximation with a slight modification, and so save a lot of work. In particular, we can use Fast Legendre Transformation.

- Due to the orthogonality of Legendre polynomials and Legendre rational functions, we provide simple implementation for this mixed spectral method.

- Benefiting from the rapid convergence of Legendre and Legendre rational approximations, we obtain very accurate numerical results even for small nodes $N$ and $M$. The numerical experiments demonstrate the high accuracy of the proposed method.

In this paper, we establish some basic results on the mixed Legendre-Legendre rational approximation, which form the mathematical foundation of the spectral method for an infinite strip. We may also consider the mixed LegendreLegendre rational interpolation which leads to the mixed Legendre-Legendre rational pseudospectral method for an infinite strip. Clearly this is preferable for actual computations.

In this paper, we use the base functions $R_{l}(x)=\frac{\sqrt{2}}{x+1} L_{l}\left(\frac{x-1}{x+1}\right)$. But we may apply the scaling base functions

$$
\widetilde{R}_{l}(x)=\frac{\sqrt{2 \beta}}{x+\beta} L_{l}\left(\frac{x-\beta}{x+\beta}\right), \quad \beta>0 .
$$

In this case, the adjustable parameter $\beta$ will offer great flexibility for matching asymptotic behaviors of the exact solutions at infinity. Furthermore, we could also consider Legendre irrational approximation or other mapped Legendre rational approximation, so that the numerical solutions fit the exact solutions more precisely in the region where the exact solutions vary rapidly. We note that the mapped Legendre approximation can also be used for bounded domains, see [30]. 


\section{REFERENCES}

[1] J. Bergh and J.Löfström, Interpolation Spaces, An Introduction, Springer-Verlag, Berlin (1976).

[2] C. Bernardi and Y. Maday, Spectral Methods, in Handbook of Numerical Analysis, Vol. 5, Techniques of Scientific Computing, 209-486, edited by P.G. Ciarlet and J.L. Lions, Elsevier, Amsterdam (1997).

[3] J.P. Boyd, Spectral method using rational basis functions on an infinite interval, J. Comp. Phys., 69 (1987), 112-142.

[4] J.P. Boyd, Orthogonal rational functions on a semi-infinite interval, J. Comp. Phys., 70 (1987), 63-88.

[5] J.P. Boyd, Chebyshev and Fourier spectral methods, 2nd edition, Dover Publication Inc., Mineda NY, (2001).

[6]C. Canuto, M.Y. Hussaini, A. Quarteroni and T.A. Zang, Spectral Methods in Fluid Dynamics, Springer, Berlin (1988).

[7] D. Funaro, Polynomial Approximations of Differential Equations, Springer-Verlag, Berlin (1992).

[8] D. Funaro and O. Kavian, Approximation of some diffusion evolution equations in unbounded domains by Hermite functions, Math. Comp., 57 (1990), 597-619.

[9] D. Gottlieb and S.A. Orszag, Numerical Analysis of Spectral Methods: Theory and Applications, SIAM-CBMS, Philadelphia (1977).

[10] Guo Ben-yu, Spectral Methods and Their Applications, World Scietific, Singapore (1998).

[11] Guo Ben-yu, Error estimation of Hermite spectral method for nonlinear partial differential equations, Math. Comp., 68 (1999), 1067-1078.

[12] Guo Ben-yu, Gegenbauer approximations and its applications to differential equations on the whole line, J. Math. Anal. and Appl., 226 (1998), 417-436.

[13] Guo Ben-yu, Jacobi spectral approximation and its applications to differential equations on the half line, J. Comp. Math., 18 (2000), 95-112.

[14] Guo Ben-yu, Gegenbauer approximations and its applications to differential equations with rough asymptotic behaviors at infinity, Appl. Numer. Math., 38 (2001), 403-425.

[15] Guo Ben-yu, Jacobi spectral method for differential equations with rough asymptotic behaviors at infinity, J. Comp. Math. Appl., 46 (2003), 95-104.

[16] Guo Ben-yu and He Li-ping, The fully discrete Legendre spectral approximation of twodimensional unsteady incompressible fluid flow in stream function form, SIAM J. Numer. Anal., 35 (1998), 146-176.

[17] Guo Ben-yu and Jie Shen, Laguerre-Galerkin method for nonlinear partial differential equations on a semi-infinite intervals, Numer. Math., 86 (2000), 635-654. 
[18] Ben-yu Guo and Jie Shen, On spectral approximations using modified Legendre rational functions: application to the Korteweg-de Vries equation on the half line, Indiana Univ. J. of Math., 50 (2001), 181-204.

[19] Ben-yu Guo, Jie Shen and Zhong-qing Wang, A rational approximation and its applications to differential equations on the half line, J. of Sci. Comp., 15 (2000), 117-148.

[20] Ben-yu Guo, Jie Shen and Zhong-qing Wang, Chebyshev rational spectral and pseudospectral methods on a semi-infinite interval, Int. J. Numer. Meth. Engng., 53 (2002), 65-84.

[21] Guo Benyu, Wang Zhongqing, Wan Zhengsu and Chu Delin, Second order Jacobi approximation with applications to fourth-order differential equations, Appl. Numer. Math., to appear.

[22] Guo Ben-yu and Xu Cheng-long, On two-dimensional incompressible fluid flow in an infinite strip, Math. Meth. Appl. Sci., 23 (2000), 1617-1636.

[23] Guo Ben-yu and Xu Cheng-long, Mixed Laguerre-Legendre pseudospectral method for incompressible flow in an infinite strip, Math. Comp., 73 (2004), 95-125.

[24] D.B. Haidvogel and T.A. Zang, The accurate solution of Possion's equation by expansion in Chebyshev polynomials, J. Comput. Phys., 30 (1979), 167-180.

[25] J.L. Lions, Quelques méthods de résolution des problèmes aux limités non linéaires, Dunod, Paris (1969).

[26] Y. Maday, B. Pernaud-Thomas and H. Vanderen, Reappraisal of Laguerre type spectral methods, La Recherche Aerospatiale, 6 (1985), 13-35.

[27] P.J. Roach, Computational fluid dynamics, 2nd ed., Hermosa, Albuquerque, NM, (1976).

[28] Jie Shen, Efficient spectral-Galerkin method I. Direct solvers of second and fourth order equations using Legendre polynomials, SIAM J. Sci. Comput., 15 (1994), 1489-1505.

[29] Jie Shen, Stable and efficient spectral methods in unbounded domains by using Laguerre functions, SIAM J. Numer. Anal., 38 (2000), 1113-1133.

[30] Jie Shen and Li-lian Wang, Error analysis for mapped Legendre spectral and pseudospectral methods, SIAM J. Numer. Anal., 42 (2004), 326-349.

[31] R. Témam, Navier-Stokes Equation, North-Holland, Amsterdam (1977).

[32] Wang Zhong-qing and Guo Ben-yu, A rational approximation and its applications to nonlinear partial differential equations on the whole line, J. Math. Anal. Appl., 274 (2002), 374-403.

[33] Xu Cheng-long and Guo Ben-yu, Mixed Laguerre-Legendre spectral method for incompressible flow in an infinite strip, Advances in Computational Mathematics, 16 (2002), 77-96. 